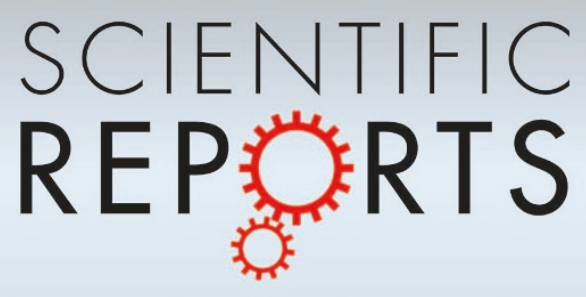

OPEN

SUBJECT AREAS:

NANOPARTICLES

COARSE-GRAINED MODELS

SELF-ASSEMBLY

Received

16 July 2014

Accepted

23 October 2014

Published

12 November 2014

Correspondence and requests for materials should be addressed to

L.-T.Y. (Ityan@mail. tsinghua.edu.cn)

\section{Predictive supracolloidal helices from patchy particles}

\author{
Ruohai Guo, Jian Mao, Xu-Ming Xie \& Li-Tang Yan
}

Key Laboratory of Advanced Materials (MOE), Department of Chemical Engineering, Tsinghua University, Beijing 100084, P. R. China.
A priori prediction of supracolloidal architectures from nanoparticle and colloidal assembly is a challenging goal in materials chemistry and physics. Despite intense research in this area, much less has been known about the predictive science of supracolloidal helices from designed building blocks. Therefore, developing conceptually new rules to construct supracolloidal architectures with predictive helicity is becoming an important and urgent task of great scientific interest. Here, inspired by biological helices, we show that the rational design of patchy arrangement and interaction can drive patchy particles to self-assemble into biomolecular mimetic supracolloidal helices. We further derive a facile design rule for encoding the target supracolloidal helices, thus opening the doors to the predictive science of these supracolloidal architectures. It is also found that kinetics and reaction pathway during the formation of supracolloidal helices offer a unique way to study supramolecular polymerization, and that well-controlled supracolloidal helices can exhibit tailorable circular dichroism effects at visible wavelengths.

T he assembly of particles with target complex structures that meet the specific applications is a challenging issue in materials science, particularly when compared with nature's ability to create highly functional structures through biomolecular self-assembly ${ }^{1,2}$. Directional interaction fields decorated on particle surface with sticky patches open up rich avenues for self-assembly into target supracolloidal structures ${ }^{3-8}$. Rational design of these building blocks for self-assembly can be significantly facilitated if the final structure can be predicted as a function of the building block parameters. The interaction fields and anisotropic shapes encoded in the building blocks allow potential approaches for such a prediction ${ }^{9-14}$. However, considering the complex energy landscape and kinetic pathway, the predictive science of sophisticated supracolloidal structures remains a key challenge. Particularly, although numerous studies on the predictive self-assembly of anisotropic particles have been reported $^{9-16}$, a priori prediction of helical supracolloidal structures from rationally designed building blocks still lacks a general roadmap and has yet to be demonstrated.

Helical structure represents the principal element responsible for the property of chirality ${ }^{17}$. Control over chirality at nano- and mesoscales is rapidly becoming a goal of great scientific interest because such unique architectures will allow optical $^{18}$, plasmonic ${ }^{19}$, and catalysis ${ }^{20}$ materials to have distinctively emergent properties ${ }^{13,14,21-25}$. This aspect is particularly relevant for photonic applications, where the optical properties are significantly influenced by the periodically arranged unit cells ${ }^{18,26}$. The notion of a directional interaction field encoded by the surface patches suggests that patchy particles can be used to generate supracolloidal helices without fixed templates that offer limited controllability and may penalize the properties of particle assemblies ${ }^{23,27}$. However, the ability to design and control supracolloidal helices assembled from patchy particles or colloids is limited by the absence of a general prediction principle. Challenges include exploring a facile design rule of patchy particles for helical self-assembly and further establishing a critical prediction principle for such supracolloidal architectures.

Inspired by the helical structures in biological macromolecules, such as DNA and proteins, we report here a new design rule of patchy particles, that we call bio-inspired design of patchy particles, for the construction of helically supracolloidal architectures through bottom-up assembly. With this conceptually new rule, we demonstrate that a vast collection of biomolecular mimetic supracolloidal helices are readily accessible. A more facile design rule of patchy particles is further derived, allowing us to establish a critical prediction principle for target supracolloidal helices. We also examine the mechanisms of supracolloidal reactions during the formation of supracolloidal helices, and demonstrate that these well-controlled and chiral supracolloidal architectures can exhibit tailored optical response.

\section{Results}

Design rules and prediction principle. To elucidate the design rules and establish the prediction principle, we carry out systematic molecular simulations of self-assembly of patchy particles consisting of a spherical core with 
decorated patches of discrete and specific interactions (see Supplementary Information and Figs. S1 and S3). To mimic the biological helices, the patchy configurations, including the position, number, and type, need to be designed based on the known structures of target biological macromolecules. For example, the patchy configuration can be designed basically according to the base pairs in DNA, and thereby these patches can direct the particles to selfassemble into a supracolloidal architecture with double-stranded helicity, replicating DNA helix at the mesoscale (Fig. 1a). Similarly, a colloidal mimetic structure of triple-stranded DNA can be formed by rationally designed patchy particles with four patches (Fig. 1b). This design rule is also useful to mimic the helical structures in proteins. As shown in Fig. 1c, a single-stranded helical supracolloidal structure similar to the $\alpha$-helix in proteins is obtained through the bottom-up assembly of patchy particles with four patches. The design rule encoded in the patchy particles is successful in self-assembling the Boerdijk-Coxeter helices which have been observed in experiments ${ }^{28}$ and previous simulations ${ }^{13}$ (Fig. 1d).

Clearly, bio-inspired design of patchy particles open up rich avenues for sophisticated self-assembly into a variety of biomolecular mimetic supracolloidal helices by controlling the arrangements and interactions of patches. The detailed patchy-configuration information of the patchy particles used in Fig. 1 has been listed in Table S1. Note that in the model two types of patches are involved, i.e., the selfcomplementary patches which can only interact with the same type of patches, and a pair of complementary patches which can only interact with each other. These patchy types have been suggested in the context of protein crystallization ${ }^{29}$ and charged patchy colloids ${ }^{30}$. Recent experimental work has also demonstrated that a significant progress has been made in the strategy for creating colloidal particles with sophisticated patchy configurations ${ }^{5-7,31}$. Moreover, we notice that the construction of supracolloidal helices presents colloidal analogs to the molecular design of some supramolecules with ordered structures (Fig. S2), which will be discussed in more detail in the section on supracolloidal reactions.

Considering the simplified and minimal model for a supracolloidal helix, we conclude from Fig. 1 that at least two types of interactions are required for cooperative and helical self-assembly: one interaction contributes to the stepwise growth of particle chains (such as P2 patches of particle 1-3 in Table S1); and the other interaction gives rise to the helicity of the self-assembled structures (such as P1 patches of particle 1-3 in Table S1). The two types of interactions lead to competing length scales which control the helical structures $^{32-34}$. Based on these findings, we derive a more facile design rule of patchy particles with significantly simplified patch configuration. As demonstrated in Fig. 2a and Particle 5 in Table S1, the green patch at the coordinate of $(0,0,1)$ is a self-complementary patchy governing the stepwise growth of particle chains, while the blue patch at $(\sin \theta, 0, \cos \theta)$ and the cyan patch at $(\sin \theta \cos \varphi, \sin \theta \sin \varphi,-\cos \theta)$ consist of a pair of complementary patches confirming the consistent and stable helicity of the supracolloidal architectures. Here, $\theta$ is the angle between $z$ direction and blue patch direction, and $\varphi$ is the angle measured from the $x$ direction to the orthogonal projection of the cyan patch direction on the $x-y$ plane. The green patch is fixed in the $z$ direction. The polar angles of the blue and cyan patches are $\theta$ and $\left(180^{\circ}-\theta\right)$ while their azimuth angles are $0^{\circ}$ and $\varphi$, respectively. It is noticed that the green patches can also be constructed by a pair of complementary patches. However, at least two types of complementary patchy particles are needed to form helices in this situation complicating the process for the designed helical superstructures.

The 3-patch particles can self-assemble into double-stranded supracolloidal helices (Figs. 2b-2d and Figs. S4-S8). The radial correlation function clarifies that the assembled helical structures are well-defined, and are of good similarity with the ideally helical model of the same values of $\theta$ and $\varphi$ (Fig. S6). Although this model may be not the minimalist model for the helical assembly, it could be the most simplified model that can lead to defined and stable supracolloidal helices which significantly reduces the possibility of the system getting into kinetic traps during the assembling process (Figs. S6 and S7). Indeed, removal of any one patch leads to more complex phase behaviors encompassing liquid and condensed phases instead of the helical phase ${ }^{13}$.

With the self-assembly of our 3-patch particles, we can build various supracolloidal helices by tuning the values of $\theta$ and $\varphi$. For instance, at $\theta=60^{\circ}$ and $\varphi=120^{\circ}$, the pitch, $p$, and radius, $r$, of the supracolloidal helix is 3.5 and 1.0, respectively (Fig. 2b and Fig.

\section{a}

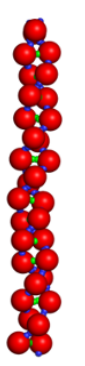

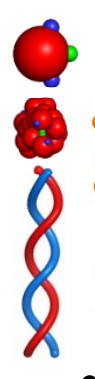

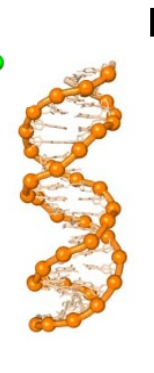

b

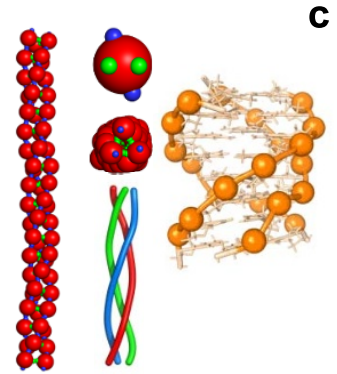

C

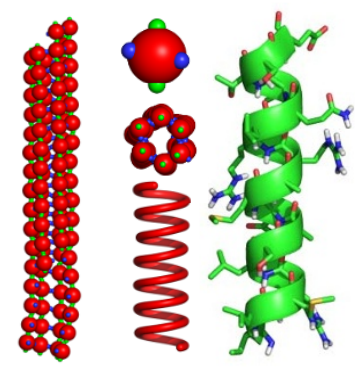

d

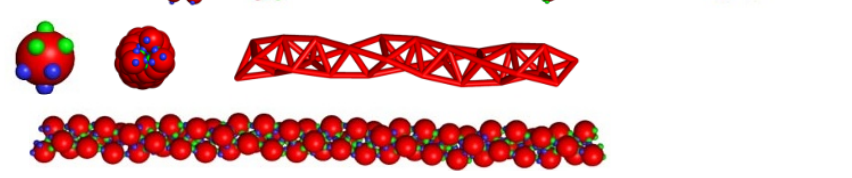

Figure 1 Biomolecular mimetic supracolloidal helices. (a), Patchy particles with three patches self-assemble into a double-stranded helix mimicking DNA double helix structure. (b), Helical structure self-assembled from patchy particles with four patches presents a colloidal mimetic structure for the triple-stranded DNA. (c), Patchy particles with four patches self-assemble into a single-stranded helically supracolloidal structure similar with the $\alpha$-helix in proteins. (d), Boerdijk-Coxeter helices formed from patchy particles with six patches. In $(\mathrm{a}-\mathrm{c})$, the left and right parts show the side view of the supracolloidal structures and the designing inspirations from the target biostructures while the top, middle, and bottom images in the middle part present the building blocks (patchy particles), the top view of helical structures, and the geometrical representation of helical structures, respectively. In (d), the left, middle, and right images in the top part show the building block, the top view of the helical structure, and the geometrical representation of the helical structure. The bottom image in (d) displays a side view of the helically supracolloidal structure. The small green and blue spheres in (a-c) represent the self-complementary patches respectively while the small green and blue spheres in (d) represent a pair of complementary patches. The detailed description of patch parameters can be found in Supplementary Information. Side and top views of each supracolloidal structure are extracted from simulated equilibrium structures. 

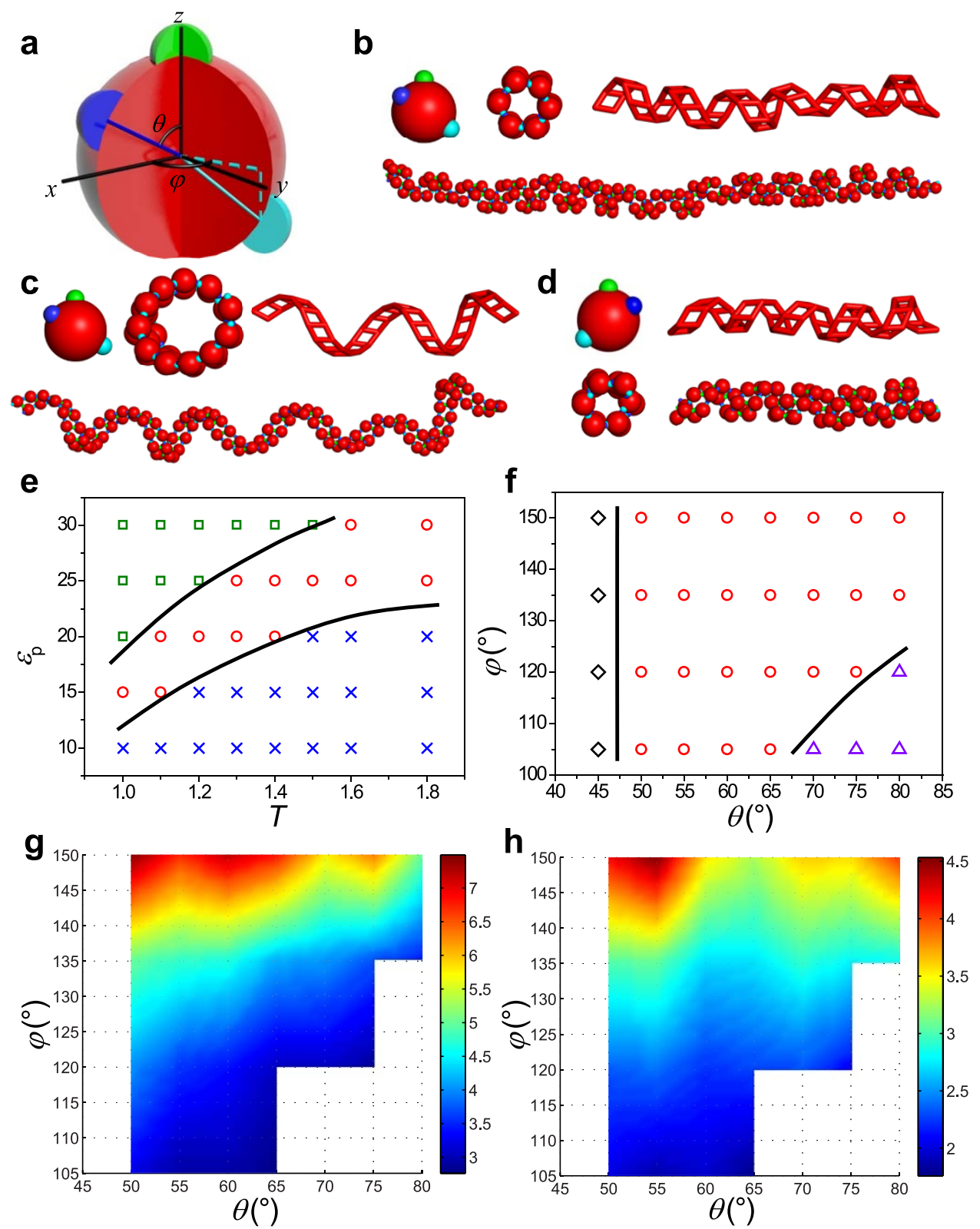

Figure 2 Tunable helical supracolloidal structures from a facile particle model. (a), Cartoon of patchy particle model used in the simulations. The green patch is a self-complementary patch while the blue patch and cyan patch is a pair of complementary patches. The relative directions of these patches are determined by angles $\theta$ and $\varphi$. (b), Right-handed double-stranded helix formed from patchy particles with patch direction of $\theta=60^{\circ}$ and $\varphi=120^{\circ}$. (c), Right-handed double-stranded helix with larger pitch and radius than those in (b) where the parameters are set as $\theta=60^{\circ}, \varphi=150^{\circ}$. $(\mathrm{d})$,

Left-handed double-stranded helix formed from patchy particles with patch direction of $\theta=60^{\circ}$ and $\varphi=-120^{\circ}$. In (b-d), the building blocks, and the top and side views of the helically supracolloidal structures and their geometrical representation are shown. (e), Phase diagram in the temperature- $\varepsilon_{P}$ space where the patch direction of particles is set as $\theta=60^{\circ}, \varphi=120^{\circ}$. Green square, red circle and blue cross denote the arrested state, the state with predominantly helical structures and the liquid state, respectively. (f), Phase diagram in the $\theta-\varphi$ space at $T=1.2$ and $\varepsilon_{P}=20$. The black diamond, red circle and purple triangle indicate the states of linear chain structure, helical structure and ring structure, respectively. (g), The average pitches of helices in the middle region of the phase diagram of (f). (h), The average diameters of helices obtained from the same patchy particles of (g).

S8). However, only increasing $\varphi$ to $150^{\circ}$ leads to a larger pitch of $p=$ 7.1 and a larger radius of $r=1.7$ (Fig. 2c and Fig. S8). Through changing the values of $\theta$ and $\varphi$, we can also control the chirality, represented by the handedness, of the supracolloidal helix. As an example, the right-handed helix of Fig. $2 \mathrm{~b}$ transits into left-handed helix of Fig. 2c where only the value of $\varphi$ is changed from $120^{\circ}$ to $-120^{\circ}$. To further evaluate the supracolloidal architectures formed by the facile model of patchy particle, we systematically simulate its assembled structures in response to different thermal conditions and structural configurations, allowing us to consider their phase behavior. Fig. $2 \mathrm{e}$ presents the phase diagram in the temperature- $\varepsilon_{P}$ space where $\varepsilon_{P}$ is the strength of the patchy interaction between the particles (see Eq.3 in Supplementary Information). A wide regime can be found in the phase diagram where equilibrium helical structures form, as denoted by the red circles. At high $\varepsilon_{P}$ and low temperature, we also observe an arrested state (Fig. S5a) while it tends to form a liquid state at low $\varepsilon_{P}$ and high temperature (Fig. S5b). Fig. $2 \mathrm{f}$ displays the other phase diagram determining the phase behaviors of the patchy particles in the $\theta-\varphi$ space. For $\theta=45^{\circ}$, the patchy particles always self-assemble into linear chains (Figs. S5c and S5e), regardless of the value of $\varphi$. When $\theta$ is no less than $70^{\circ}$ and $\varphi$ is no larger than $120^{\circ}$, a phase of ring structure can be identified (Figs. S5d and S5f). In 

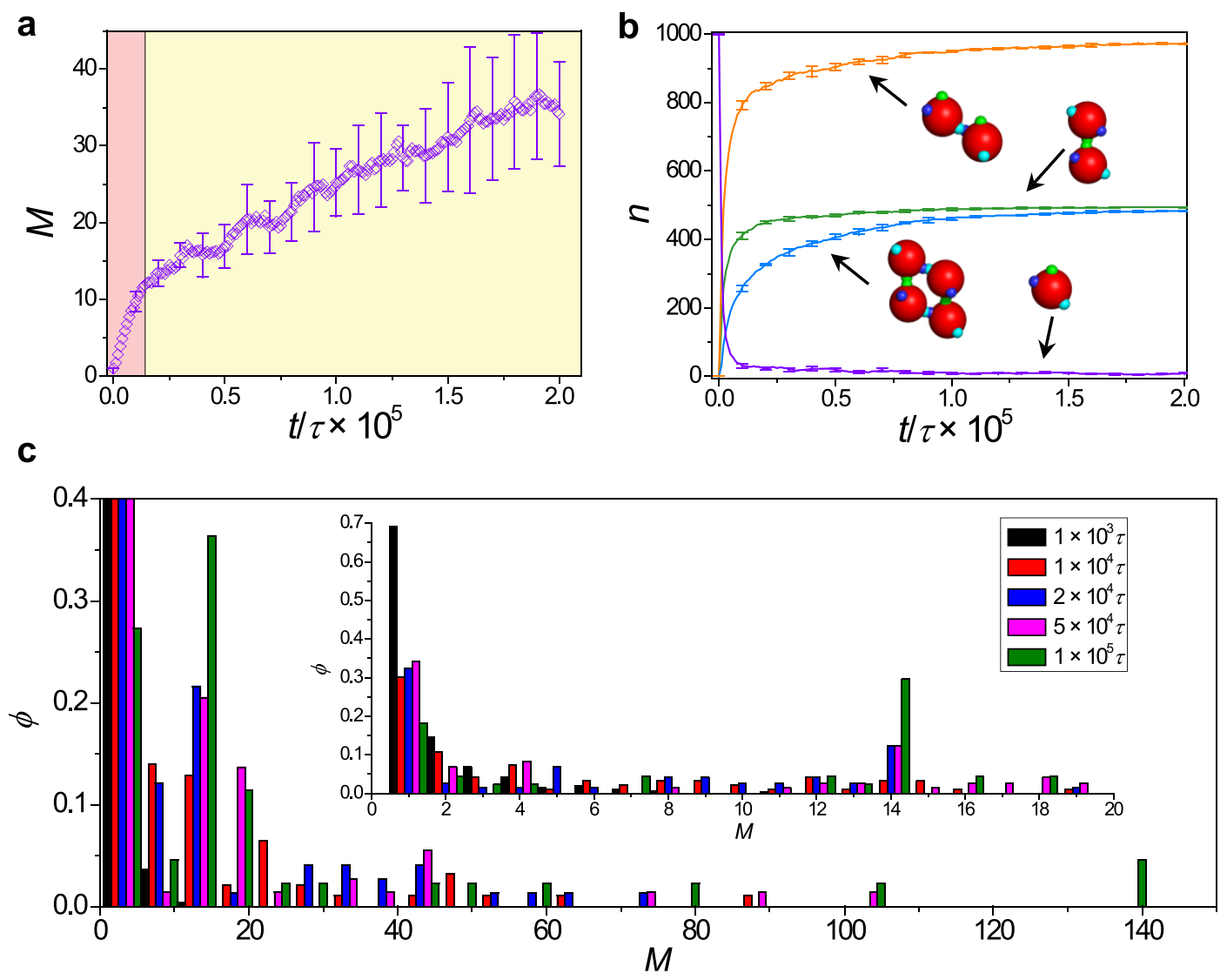

Figure 3 Kinetics in the self-assembling process of the helically supracolloidal structure. (a), The number averaged degree of colloidal clusters as a function of self-assembling time $t$. (b), Variation in the number of patch groups in the course of self-assembly for species shown in inserts. (c), Number averaged distributions of colloidal clusters at different times. Error bars in (a) and (b) indicate standard deviation. Systems of patchy particles with $\theta=60^{\circ}$ and $\varphi=120^{\circ}$ are chosen to show the kinetics process.

both these phase regimes, the patchy configurations induce significantly spatial confinement preventing the formation of the helical structures. This underscores the importance of surface configurations in the supercolloidal construction from these model particles.

Fig. $2 \mathrm{f}$ shows that a phase of equilibrium helical structure can be found within large ranges of $\theta$ and $\varphi$, offering a broad space for the control of the helically supracolloidal architectures. For better understanding the relationship between the geometrical parameters of supracolloidal helices and patchy configurations, we calculate the pitch and diameter of the helix at each point in the helix regime of Fig. $2 \mathrm{f}$ (denoted by the red circles). The calculation results are summarized in the images of Figs. $2 \mathrm{~g}$ and $2 \mathrm{~h}$, where the color bars indicate the values of these parameters. Fig. $2 \mathrm{~g}$ shows that the pitch of the supracolloidal helices increases from about 3.0 to more than 7.0 when $\varphi$ is increased from $105^{\circ}$ to $150^{\circ}$, while it is reduced for a larger $\theta$. Increasing $\varphi$ from $105^{\circ}$ to $150^{\circ}$ results in the increase of the helix diameter from about 1.0 to 4.5 although the value of $\theta$ has a slight effect on the diameter of the helix (Fig. 2h). These results highlight the possibility of $\theta$ and $\varphi$ in the prediction of helically supracolloidal architectures from the patchy particles.

An ideal helical structure can be obtained from the model patchy particles with a certain patchy configuration, where the corresponding patchy pairs are set to be perfectly linked. To further examine the ability of $\theta$ and $\varphi$ in the prediction, we compare the pitch and radius of the helical structures from simulations and those from the ideally helical structures. The pitch and radius of the ideal helix can be calculated as $p=b_{0} \cos \theta \cdot 360^{\circ} /\left(180^{\circ}-\varphi\right)$ and $r=b_{0} \sin \theta /$ $[2 \cos (\varphi / 2)]$, respectively, where $b_{0}=1.16$ is the average distance of patchy pairs in the simulations. As shown in Fig. S8, the values and varying trends of the pitch and radius with various $\theta$ and $\varphi$ are very similar for both these cases. This good agreement between simulation results and ideal model confirms that $\theta$ and $\varphi$ are effective approaches to predict the helically supracolloidal architectures from the patchy particles, such as their pitch, radius, and handedness. Taken together, the patchy-particle model proposed here not only leads to a facile and novel design rule to construct supracolloidal helices but also allows us to establish a critical prediction principle for supracolloidal architectures.

Mechanisms of supracolloidal reactions. The self-assembly of patchy particles into a target structure can be viewed as a "supracolloidal reaction" which bears a strong similarity to polymerization reaction and can provide insight into molecular design and mechanisms of chemical reactions ${ }^{28,35,36}$. In this context, we note that the construction of supracolloidal helices presents colloidal analogs of the molecular design to some supramolecules with ordered structures ${ }^{37,38}$ (Fig. S2). The patchy particles act as multifunctional monomer units that "react" with each other, in a process analogous to supramolecular polymerization ${ }^{36-38}$. As shown in Fig. S2, the intermolecular hydrogen bonds, $\pi-\pi$ interactions, and metal-ligand binding contribute to the functions resembling patches. The multi-patchy configuration significantly increases the sophistication of kinetics and reaction pathway. Fig. 3a shows the time dependence of the average number of patchy particles in the supracolloidal chains, $M$, that resembles the number-average degree of polymerization. Interestingly, the $M \sim t$ plot can be divided into two regimes. In the first regime, $M$ increases linearly with time, being characteristic of reaction-controlled step-growth polymerization. However, the linearity of the $M \sim t$ plot is relatively suppressed and the plot presents obvious fluctuation in the second regime. 
To understand the kinetic mechanisms, we turn to the detailed analysis of the change in the concentrations of different species in the course of self-assembly. Based on patchy interactions directed by their types, four representative species are concerned: individual patchy particle (IP), colloidal cluster linked by self-complementary patches (SP), colloidal cluster linked by a pair of complementary patches (PP), and the elementary colloidal cluster of perfect helix (EC) (Fig. 3b). Fig. 3b shows that the concentration of IP is extremely reduced at the initial stage owing to the formation of SP and PP. The complementary patches in SP and PP link each other spontaneously while the formation of EC with long-ranged patchy addition is relatively slow. Thus, the reactions of SP and PP dominate the kinetics in the first regime, resulting in the characteristic of reaction-controlled step-growth polymerization. In the second regime, the reaction of EC however becomes the major effect, where sophisticated dynamical interconversion between clusters, which is elaborated in Fig. 4 and Fig. S9, induces obvious fluctuation in the kinetic plot. The distribution of colloidal clusters also becomes wider in the second regime, as demonstrated by Fig. 3c.

Fig. 4 summarizes all the reaction pathways that we can observe in the formation of supracolloidal helix. The pathways form a complex network in which multiple cluster possibilities emanate from the addition of complementary patches at the initial stage. The directional addition of complementary patches however becomes relatively difficult when long-ranged structures occur. This leads to complex mechanism of dynamical interconversion between clusters. The major mechanisms include step-by-step addition of individual particles, addition of fragments, cyclization of fragments, break of incomplete rings, fracture and reconfiguration of disordered clusters, and addition and reconfiguration of helices. These mechanisms have been elucidated exhaustively in Supplementary Information and Figs. S10-S12. In general, three distinctive characteristics can be derived from these mechanisms: the first is that all the kinetic process starts from the patchy addition which leads to the colloidal analogs of step-growth polymerization at the preliminary stage; the second is that the structural reconfiguration plays a key role in the formation of perfect helix because it facilitates a patch to find its couple; Finally, the cyclization reaction and structural reconfiguration can induce the formation of stable colloidal rings consisting of 14 patchy particles, which can also be identified from the peak at $M=14$ in the number averaged distributions of colloidal clusters (Fig. $3 \mathrm{c}$ and its inset). These findings may provide fundamental information for understanding the mechanisms of some reactions in supramolecules chemistry towards helically molecular architectures ${ }^{37-40}$.

Optical response. Circular dichroism (CD) effects in the visible spectrum have been predicted to occur through collective Coulomb interaction of plasmonic dipoles in chiral assemblies of metal nanoparticles ${ }^{22,23,27}$. As an example, by defining a patchy particle as a gold particle with a diameter of $10 \mathrm{~nm}$, we consider the CD response of supracolloidal helices assembled from our model patchy particles (see Supplementary Information for calculation details). To identify the effects of patchy configuration on CDs, representative sections are extracted from right-handed helical supracolloidal chains, where the particle number of each section is fixed at 24 . Figs. $5 \mathrm{a}$ and $5 \mathrm{~b}$ show the helical sections at various $\varphi$ and $\theta$, and their corresponding CDs, with a characteristic bisignate peak-dip shape, are summarized in Figs. $5 \mathrm{c}$ and $5 \mathrm{~d}$, respectively. The calculation for ideally helical structures clarifies that a larger pitch leads to a stronger and red-shifted CD signal while a larger radius corresponds to a weaker and red-shifted CD signal (Fig. S13). As demonstrated in Figs. 2 and S8, increasing $\varphi$ results in the increase of both pitch and radius of the helical structures. Therefore, an obvious red-shift can be identified from the CDs of Fig. $5 c$ whereas the changing trend of signal strength is irregular owing to its inverse dependences on radius and pitch. Figs. 2 and S8 also demonstrate that a larger $\theta$ can reduce pitch whereas its effect on radius is relatively trivial. Increasing $\theta$ thereby leads to weaker and blue-shifted signal, as shown in Fig. 5d. It is also not difficult to anticipate that vertically mirrored dip-peak signals can be obtained by changing the handedness of the supracolloidal helices as indicated in Figs. $2 \mathrm{~b}$ and $2 \mathrm{~d}^{27}$. In this context, the optical response of our supracolloidal helices is rationally designed and tailorable in color, intensity, and handedness.

\section{Discussion}

Our results provide an important step toward a predictive science of supracolloidal helices assembled from designed building blocks, which significantly prompts experimental realization of colloidal crystal and metamaterials with controllable helicity. We expect that our facile design model of patchy particles will be necessary to guide experiments because the experimental implementation of particles with sophisticated patchy configurations is now becoming available ${ }^{5-7,31}$. The bio-inspired design of patchy particles offers a unique approach to understand the formation mechanism and functionality of helical structures ubiquitous in nature, such as DNA, proteins, and virus. In view of colloidal analogs of the reaction kinetics of supracolloidal helices to some supramolecular polymerization, the kinetics and detailed reaction pathways during the particle assembly can provide fundamental insight into the kinetic mechanism of supramolecules chemistry towards helically molecular architectures, although the quantitative correlation between them needs to be further developed in the future. Finally, the tailored optical response of supracolloidal helices suggests their important applications in the design of optical devices with desired properties.

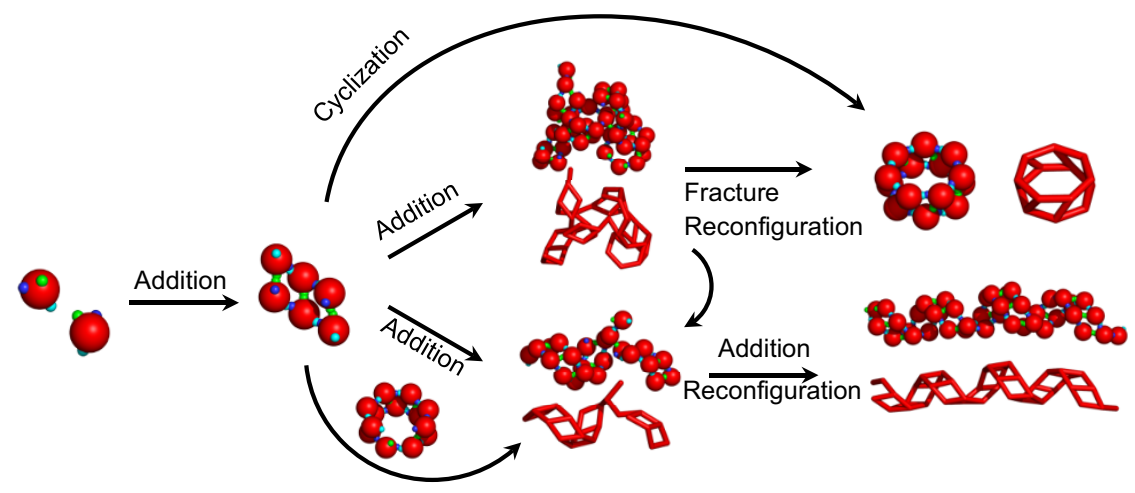

Figure 4 | Reaction pathways of helical self-assembly. The mechanisms, all of which we have observed in simulations, include addition of individual particles, addition of fragments, cyclization of fragments, break of incomplete rings, fracture and reconfiguration of disordered clusters, and addition and reconfiguration of helices. Details of the structure evolution are shown in Figs. S10-S12. 

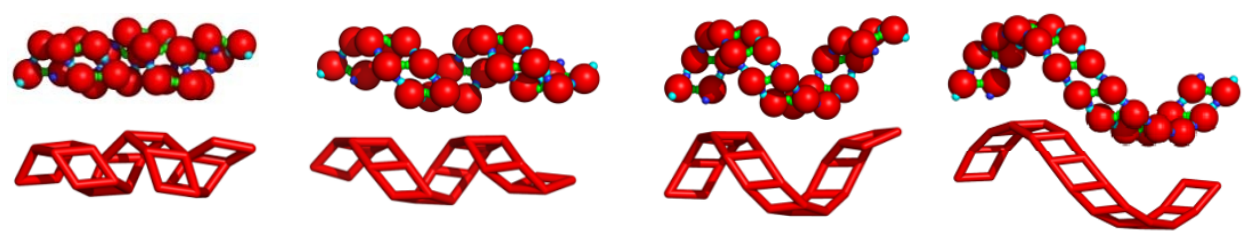

b
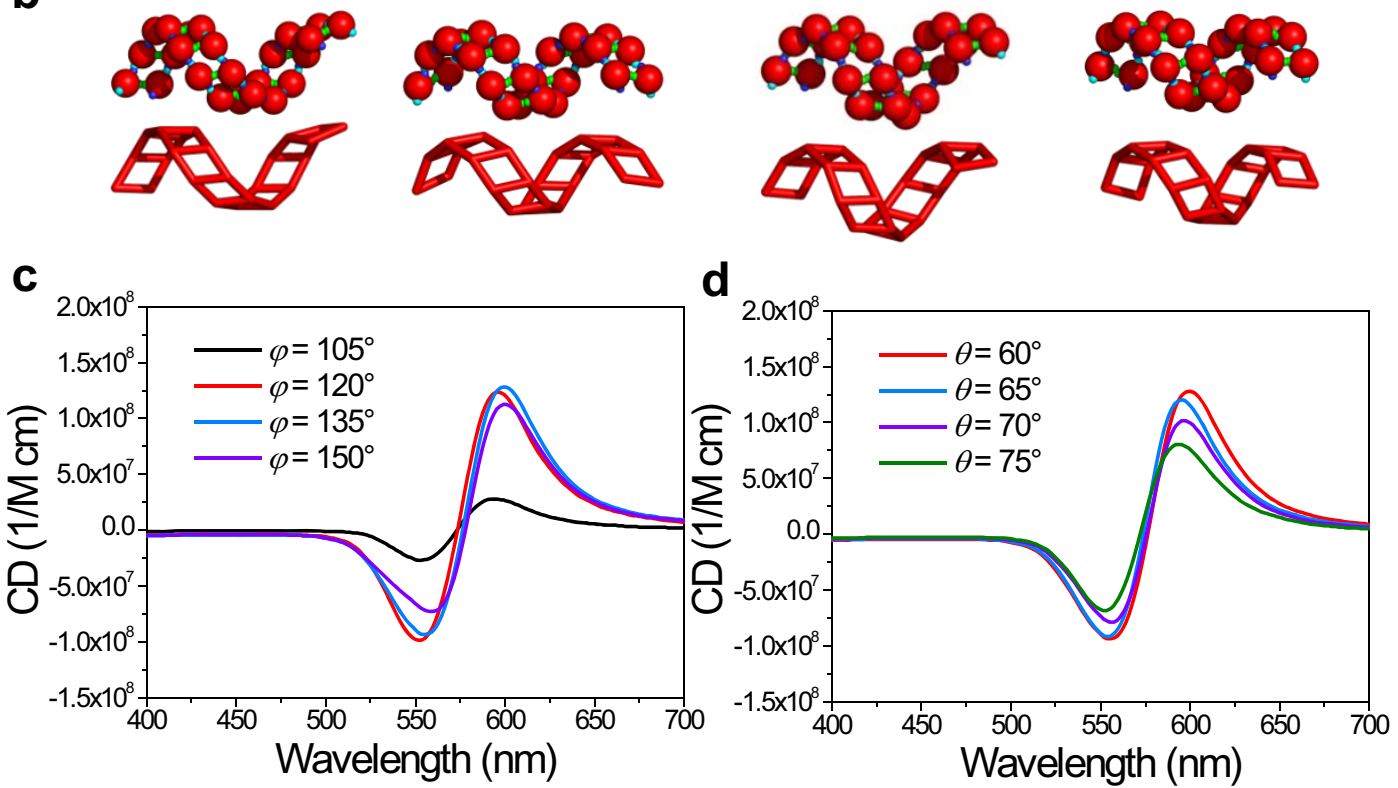

Figure 5 Tailored optical response of self-assembled supracolloidal helices. (a), Representative sections extracted from simulated helical structures for patchy particles with $\theta=60^{\circ}$ and $\varphi=105^{\circ}, 120^{\circ}, 135^{\circ}, 150^{\circ}$ (from left to right). (b), Representative sections extracted from simulated helical structures for patchy particles with $\varphi=135^{\circ}$ and $\theta=60^{\circ}, 65^{\circ}, 70^{\circ}, 75^{\circ}$ (from left to right). (c), The theoretically predicted circular dichroisms (CDs) for the geometries in (a). (d), The theoretically predicted CDs for the geometries in (b).

\section{Methods}

To determine the phase behavior for each type of patchy particle, molecular dynamics with a Langevin thermostat at constant temperature are carried out. The patch character is described using the model proposed by Morgan et al. ${ }^{13}$ and the particles interact via an anisotropic interaction potential that depends on both the separation between particle surfaces and the orientations of patch axes. The interparticle forces and torques are derived from the pair potentials between particles using a previous method ${ }^{41}$ and the rotational degrees of freedom of the particles are incorporated using the equations for rotation of rigid bodies with quaternions ${ }^{42}$. The motion of patchy particles are updated though the velocity Verlet scheme with a time step $\Delta t=0.001$. To account for finite size effects, we simulate systems of 300, 1000 and 2000 particles for each of the patchy particles. Each simulation is equilibrated for a minimum of $5 \times 10^{8}$ steps and the equilibration of systems is confirmed through examining the system potential energy. Actually, we find that in most of the runs the fluctuations in the system potential are small and the morphology does not substantially change after $2 \times 10^{8}$ steps. To construct the phase diagram and investigate the dynamics of helical formation, at least 3 independent runs are performed for each system.

The $\mathrm{CD}$ responses of assembled structures are computed using a numerical too called DDSCAT, which was developed by Draine and Flatau ${ }^{43}$. To illustrate the influence of structure on the optical response, here we set the building blocks in the helices as gold particles with a diameter of $10 \mathrm{~nm}$. It should be emphasized that this particular assumption for the particle size only provides an example for the following calculation. A short helix randomly extracted from self-assembled structure is simulated and the wavelength is set in the visible band of $400 \mathrm{~nm} \sim 700 \mathrm{~nm}$ accounting for the collective dipole-dipole interactions between particles. The short helix consists of 24 particles which comprise at least one helical period. We believe this method is capable to predict the $\mathrm{CD}$ responses of self-assembled structures since previous simulations have shown that in general the shift saturates at around 10 particles with increasing length of the one-dimensional chain of particles ${ }^{44}$. More details regarding the methods can be found in Supplementary Information.

1. Whitesides, G. M. \& Grzybowski, B. Self-assembly at all scales. Science 295, 2418-2421 (2002).

2. Frenkel, D. Playing tricks with designer "atoms" Science 296, 65-66 (2002).

3. Glotzer, S. C. \& Solomon, M. Anisotropy of building blocks and their assembly into complex structures. Nat. Mater. 6, 557-562 (2007).

4. Zhang, Z. \& Glotzer, S. C. Self-assembly of patchy particles. Nano Lett. 4 , $1407-1413$ (2004).
5. Chen, Q., Bae, S. C. \& Granick, S. Directed self-assembly of a colloidal kagome lattice. Nature 469, 381-384 (2011).

6. Wang, Y. et al. Colloids with valence and specific directional bonding. Nature 491, 51-55 (2012)

7. Walker, D. A., Leitsch, E. K., Nap, R. J., Szleifer, I. \& Grzybowski, B. Geometric curvature controls the chemical patchiness and self-assembly of nanoparticles. Nat. Nanotechnol. 8, 676-681 (2013).

8. DeVries, G. A. et al. Divalent metal nanoparticles. Science 315, 358-361 (2007).

9. Damasceno, P. F., Engel, M. \& Glotzer, S. C. Predictive self-assembly of polyhedra into complex structures. Science 337, 453-457 (2012).

10. Escobedo, F. A. \& Agarwal, U. Mesophase behaviour of polyhedral particles. Nat. Mater. 10, 230-235 (2011).

11. Ramano, F. \& Sciortino, F. Patterning symmetry in the rational design of colloidal crystals. Nat. Commun. 3, 975-980; DOI:10.1038/ncomms1968 (2012).

12. Gang, O. \& Zhang, Y. Shaping phases by phasing shapes. ACS Nano 5, 8459-8465 (2011).

13. Morgan, J. W. R., Chakrabarti, D., Dorsaz, N. \& Wales, D. J. Designing a Bernal spiral from patchy colloids. ACS Nano 7, 1246-1256 (2013).

14. Nguyen, T. D. \& Glotzer, S. C. Switchable helical structures formed by the hierarchical self-assembly of laterally tethered nanorods. Small 18, 2092-2098 (2009).

15. Maritan, A., Micheletti, C., Trovato, A. \& Banavar, J. R. Optimal shapes of compact strings. Nature 406, 287-290 (2000).

16. Micheletti, C., Banavar, J. R. \& Maritan, A. Conformations of proteins in equilibrium. Phys. Rev. Lett. 87, 088102 (2001).

17. Harris, A. B., Kamien, R. D. \& Lubensky, T. C. Molecular chirality and chiral parameters. Rev. Mod. Phys. 71, 174-1757 (1999).

18. Gansel, J. K. et al. Gold helix photonic metamaterial as broadband circular polarizer. Science 325, 1513-1515 (2009).

19. Valev, V. K., Baumberg, J. J., Sibilia, C. \& Verbiest, T. Chirality and chiroptical effects in plasmonic nanostructures: fundamentals, recent progress, and outlook. Adv. Mater. 25, 2517-2534 (2013).

20. Inoue, Y. \& Ramamurthy, V. Chiral Photochemistry (Marcel Dekker, New York, 2004).

21. Yin, Y. \& Xia, Y. Self-assembly of spherical colloids into helical chains with wellcontrolled handedness. J. Am. Chem. Soc. 125, 2048-2049 (2003).

22. Kuzyk, A. et al. DNA-based self-assembly of chiral plasmonic nanostructures with tailored optical response. Nature 483, 311-314 (2012). 
23. Song, C. et al. Tailorable plasmonic circular dichroism properties of helical nanoparticles superstructures. Nano Lett. 13, 3256-3261 (2013).

24. Gibaud, T. et al. Reconfigurable self-assembly through chiral control of interfacial tension. Nature 481, 348-351 (2012).

25. Olesen, S. W., Fejer, S. N., Chakrabarti, D. \& Wales, D. J. A left-handed building block self-assembles into right- and left-handed helices. RSC Adv. 3, 12905-12908 (2013).

26. Soukoulis, C. M. \& Wegener, M. Past achievements and future challenges in the development of three-dimensional photonic metamaterials. Nat. Photonics $\mathbf{5}$, 523-530 (2011).

27. Fan, Z. \& Govorov, A. O. Plasmonic circular dichroism of chiral metal nanoparticle assemblies. Nano Lett. 10, 2580-2587 (2010).

28. Chen, Q. et al. Supracolloidal reaction kinetics of Janus spheres. Science 331 199-202 (2011).

29. Hloucha, M., Lodge, J. F. M., Lenhoff, A. M. \& Sandler, S. I. A patch-antipatch representation of specific protein interactions. J. Cryst. Growth 232, 195-203 (2001).

30. Doye, J. P. K. et al. Controlling crystallization and its absence: proteins colloids and patchy models. Phys. Chem. Chem. Phys. 9, 2197-2205 (2007).

31. He, Z. \& Kretzschmar, I. Template-assisted GLAD: approach to single and multipatchy patchy particles with controlled patch shape. Langmuir 29, 15755-15761 (2013)

32. Chakrabarti, D., Fejer, S. N. \& Wales, D. J. Rational design of helical architectures. Proc. Natl. Acad. Sci. U.S.A. 106, $20164-20167$ (2009).

33. Fejer, S. N., Chakrabarti, D. \& Wales, D. J. Self-assembly of anisotropic particles. Soft Matter 7, 3553-3564 (2011).

34. Forman, C. J., Fejer, S. N., Chakrabarti, D., Barker, P. D. \& Wales, D. J. Local frustration determines molecular and macroscopic helix structures. J. Phys. Chem. B 117, 7918-7928 (2013).

35. Liu, K. et al. Step-growth polymerization of inorganic nanoparticles. Science 329, 197-200 (2010).

36. Klinkova, A., Therien-Aubin, H., Choueiri, R. M., Rubinstein, M. \& Kumacheva, E. Colloidal analogs of molecular chain stoppers. Proc. Natl. Acad. Sci. U.S.A. 110, 18775-18779 (2013)

37. van Gorp, J. J., Vekemans, J. \& Meijer, E. W. $C_{3}$-symmetrical supramolecular architectures: Fibers and organic gels from discotic trisamides and trisureas. J. Am. Chem. Soc. 124, 14759-14769 (2002).

38. Kim, H. J., Zin, W. C. \& Lee, M. Anion-directed self-assembly of coordination polymer into tunable secondary structure. J. Am. Chem. Soc. 126, 7009-7014 (2004).

39. Ho, R. M., Chiang, Y. W., Lin, S. C. \& Chen, C. K. Helical architectures from selfassembly of chiral polymers and block copolymers. Prog. Polym. Sci. 36, 376-453 (2011)
40. Korevaar, P. A. et al. Pathway complexity in supramolecular polymerization. Nature 481, 492-496 (2012).

41. Allen, M. P. \& Germano, G. Expressions for forces and torques in molecular simulations using rigid bodies. Mol. Phys. 104, 3225-3235 (2006).

42. Miller, T. F. et al. Symplectic quaternion scheme for biophysical molecular dynamics. J. Chem. Phys. 116, 8649-8659 (2002).

43. Draine, B. T. \& Flatau, P. J. Discrete dipole approximation for scattering calculation. J. Opt. Soc. Am. A 11, 1491-1499 (1994).

44. Arnold, M. D., Blaber, M. G., Ford, M. J. \& Harris, N. Universal scaling of local plasmons in chains of metal spheres. Opt. Express 18, 7528-7542 (2010).

\section{Acknowledgments}

We thank B. Dong, and Z. Huang for helpful discussions. This work is supported by the National Natural Science Foundation of China under grant nos.51273105, 21174080 and 21422403

\section{Author contributions}

L.T.Y. inspired the research. R.G. and L.T.Y. designed and performed the simulations. R.G., J.M. and L.T.Y. analyzed the data. X.X.M. gave scientific advice. All authors discussed and co-wrote the paper.

\section{Additional information}

Supplementary information accompanies this paper at http://www.nature.com/ scientificreports

Competing financial interests: The authors declare no competing financial interests.

How to cite this article: Guo, R., Mao, J., Xie, X.-M. \& Yan, L.-T. Predictive supracolloidal helices from patchy particles. Sci. Rep. 4, 7021; DOI:10.1038/srep07021 (2014).

(c) (i) (-) $\odot$ This work is licensed under a Creative Commons Attribution-NonCommercialNoDerivs 4.0 International License. The images or other third party material in this article are included in the article's Creative Commons license, unless indicated otherwise in the credit line; if the material is not included under the Creative Commons license, users will need to obtain permission from the license holder in order to reproduce the material. To view a copy of this license, visit http:// creativecommons.org/licenses/by-nc-nd/4.0/ 